\title{
The clinical impact of nucleic acid amplification tests on the diagnosis and management of tuberculosis in a British hospital
}

\author{
M Taegtmeyer, ${ }^{1} \mathrm{~N} J$ Beeching, ${ }^{1} \mathrm{~J}$ Scott,,${ }^{2} \mathrm{~K}$ Seddon, ${ }^{2} \mathrm{~S}$ Jamieson, ${ }^{3}$ S B Squire, \\ H C Mwandumba, ${ }^{1}$ A R 0 Miller, ${ }^{1}$ P D 0 Davies, ${ }^{3}$ C M Parry ${ }^{2}$
}

${ }^{1}$ Tropical and Infectious Diseases Unit, Royal Liverpool University Hospital, Liverpool, UK; ${ }^{2}$ Department of Medical Microbiology, Royal Liverpool University Hospital, Liverpool, UK; ${ }^{3}$ Tuberculosis Research Unit, Cardiothoracic Centre, Liverpool, UK

Correspondence to: Dr M Taegtmeyer, Tropical and Infectious Diseases Unit, Royal Liverpool University Hospital, Prescot Street, Liverpool L7 8XP, UK; M.Taegtmeyer@liverpool. ac.uk

Received 17 May 2007 Accepted 9 October 2007 Published Online First 5 December 2007

\begin{abstract}
Background: Nucleic acid amplification tests (NAAT) based on PCR provide rapid identification of Mycobacterium tuberculosis and the detection of rifampicin resistance. Indications for their use in clinical samples are now included in British tuberculosis guidelines.
\end{abstract}

Methods: A retrospective audit of patients with suspected mycobacterial infection in a Liverpool hospital between 2002 and 2006. Documentation of the impact of NAAT usage in acid fast bacillus (AFB) microscopy positive samples on clinical practice and the influence of a multidisciplinary group on their appropriate use, compared with British guidelines.

Results: Mycobacteria were seen or isolated from 282 patients and identified as $M$ tuberculosis in 181 (64\%). NAAT were indicated in 87/123 AFB positive samples and performed in 51 (59\%). M tuberculosis was confirmed or excluded by this method in $86 \%$ of tested samples within 2 weeks, compared with $7 \%$ identified using standard methods. The appropriate use of NAAT increased significantly over the study period. The NAAT result had a clinical impact in 20/51 (39\%) tested patients. Culture results suggest the potential for a direct clinical impact in $8 / 36(22 \%)$ patients in which it was indicated but not sent and 5/36 (14\%) patients for whom it was not indicated. Patients managed by the multidisciplinary group had a higher rate of HIV testing and appropriate use of NAAT.

Conclusions: There were significant clinical benefits from the use of nucleic acid amplification tests in this low prevalence setting. Our data suggest that there would be additional benefit from their use with all AFB smear positive clinical samples.

Tuberculosis (TB) is an increasing problem in the $\mathrm{UK}^{1}$ and prompt diagnosis improves outcome and facilitates appropriate infection control and public health actions. ${ }^{2}$ Mycobacterium tuberculosis (MTB) and environmental mycobacteria have a similar microscopic appearance and molecular tests that provide rapid identification are increasingly used in clinical practice. ${ }^{34}$ British guidelines ${ }^{2}{ }^{5}$ recommend the use of nucleic acid amplification tests (NAAT) in acid fast bacillus (AFB) smear positive samples if rapid confirmation of $T B$ diagnosis would alter the care of the patient, or before conducting a large contact-tracing initiative.

Global concerns about rising rates of multidrug resistant (MDR) and extensively drug resistant strains of $\mathrm{MTB}^{67}$ mean that technologies allowing early detection of resistance are increasingly important. As rifampicin resistance is commonly due to mutations in a single gene (the rpoB gene), this can also be detected using a NAAT method. ${ }^{89}$ Although the currently available tests for rpoB mutations do not detect all cases of rifampicin resistance, and will not detect isolated isoniazid resistance, the association between rifampicin resistance and MDR-TB is strong, with one report showing $95 \%$ of rifampicin resistant strains to be associated with resistance to isoniazid. ${ }^{10}$ British guidelines therefore also advocate use of rapid diagnostic tests for rifampicin resistance if a risk assessment suggests a patient might have MDRTB. Detection of rifampicin resistance by NAAT is taken to indicate MDR-TB until full sensitivity profiles become available.

Liverpool has a low prevalence but a rising incidence of TB, with 47 cases notified in 2002 and 86 in 2005, out of a population of approximately 650000 which has changed little in that time period. ${ }^{11}$ The recent increases reflect national trends ${ }^{12}$ and coincide with Liverpool being designated as a "dispersal" centre for refugees in $2002 .^{13}$

We conducted a retrospective analysis of the clinical impact of the use of NAAT in patients with suspected TB, who had a clinical sample that was AFB smear positive between 2002 and 2006, and we examined the influence of a multidisciplinary approach on the appropriate use of investigations in this low prevalence setting.

\section{METHODS}

The Department of Microbiology of the Royal Liverpool University Hospital receives samples for TB diagnosis from inpatient wards, from a chest clinic that undertakes $\mathrm{TB}$ screening, from the Tropical and Infectious Diseases Unit and from some general practitioners (family physicians). A multidisciplinary group was convened in 2003 to improve the management and coordination of care of patients with TB. This group includes infectious disease and chest clinicians as well as clinical microbiologists and TB specialist community nurses. In a weekly meeting, the group reviews patients with suspected TB and patients on treatment with active problems, including decisions about sending samples for NAAT. It had been the policy of the tropical and infectious disease unit for at least 7 years, and more recently of the multidisciplinary team, to request HIV antibody testing for all patients with suspected or proven mycobacterial infection, unless the patient refuses consent. Timing of HIV antibody testing related to 
Table 1 Mycobacterial isolates and usage of nucleic acid amplification tests

\begin{tabular}{|c|c|c|c|c|c|c|}
\hline \multirow[b]{3}{*}{ Isolate } & \multirow[b]{3}{*}{ Total } & \multicolumn{4}{|c|}{ AFB smear positive } & \multirow{3}{*}{$\begin{array}{l}\begin{array}{l}\text { AFB smear } \\
\text { negative }\end{array} \\
\text { Total }\end{array}$} \\
\hline & & \multirow[b]{2}{*}{ Total } & \multicolumn{2}{|c|}{ NAAT indicated } & \multirow{2}{*}{$\begin{array}{l}\text { NAAT } \\
\text { not } \\
\text { indicated }\end{array}$} & \\
\hline & & & Sent & Not sent & & \\
\hline M tuberculosis complex (MDR isolate) & $180(8)$ & $98(4)$ & $47(3)$ & $32(1)$ & $19(0)$ & $82(4)$ \\
\hline M avium complex & 30 & 5 & 0 & 1 & 4 & 25 \\
\hline M gordonae & 29 & 4 & 2 & 0 & 2 & 25 \\
\hline M kansasii & 16 & 8 & 1 & 3 & 4 & 8 \\
\hline Other & 25 & 6 & 0 & 0 & 6 & 19 \\
\hline Culture negative & 2 & 2 & 1 & 0 & 1 & 0 \\
\hline Total & 282 & 123 & 51 & 36 & 36 & 159 \\
\hline
\end{tabular}

${ }^{*}$ Multidrug resistant isolate (resistant to rifampicin and isoniazid).

$\dagger M$ xenopi (6), $M$ malmoense (5), $M$ chelonae (4), $M$ abscessus (3), $M$ marinum (1), $M$ lentiflavum (1), $M$ szulgai (1), environmental Mycobacteria not speciated (4).

AFB, acid fast bacillus; MDR, multidrug resistant; NAAT, nucleic acid amplification tests.

the time of availability of mycobacterial tests was not recorded for the purposes of this study. British guidelines have recommended HIV testing of patients with TB since the year $2000 .^{5}$

Sputum and bronchial lavage samples obtained from patients with suspected mycobacterial infection were routinely treated with dithiothreitol (Sputasol, Oxoid, Basingstoke, UK), centrifuged and the deposit used to make a smear. For samples from sterile sites, such as fluids or tissues, a direct smear was made. All smears were then examined by auramine-phenol stain using fluorescence microscopy. Non-sterile samples were decontaminated with $4 \%$ sodium hydroxide and concentrated by centrifugation. The decontaminated non-sterile samples and all sterile samples were inoculated into MBBacT bottles containing liquid media for mycobacterial culture and incubated in an automated system for 6-10 weeks (Organon Teknika, Dublin, Ireland). Culture positive samples were submitted to the Regional Centre of Mycobacteriology, Newcastle, UK for identification and sensitivity testing. MDR isolates of MTB were defined as those resistant to rifampicin and isoniazid. ${ }^{6}$

If the sample was AFB smear positive sputum, a fresh undecontaminated sample was requested and sent to the United Kingdom Health Protection Agency Mycobacterial Reference Unit who performed NAAT using the INNO-LiPA Rif.TB assay (Immunogenetics, Zwijndrecht, Belgium). ${ }^{14}$ Samples were sent if rapid confirmation of MTB would

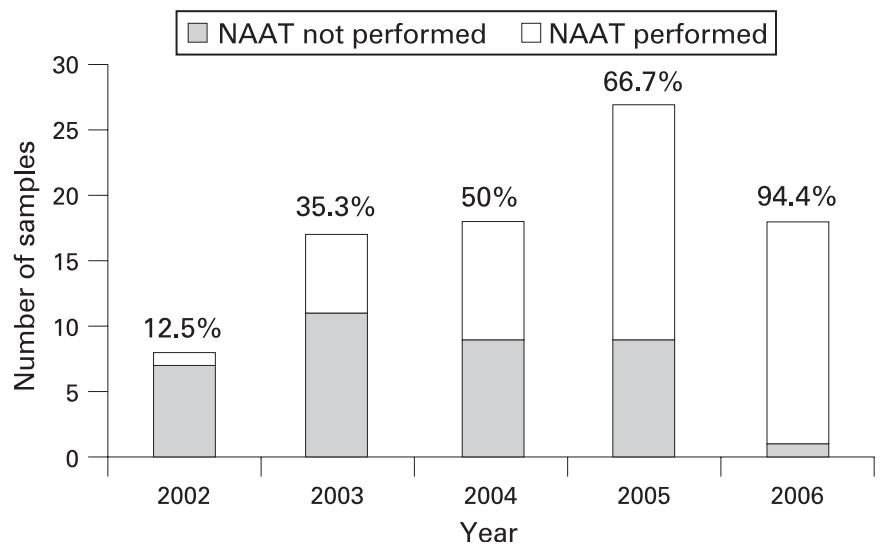

Figure 1 Use of nucleic acid amplification tests (NAAT) between 2002 and 2006 in 87 acid fast bacillus smear positive samples for which their use was indicated by British guidelines. ${ }^{2}$ Percentages indicate the proportion of samples with NAAT performed each year. influence clinical management (by exclusion of non-TB mycobacteria in HIV positive or otherwise immunocompromised patients); if there were concerns about possible drug resistance, including MDR-TB (previous TB treatment, contact with MDR-TB, a patient from a TB endemic area); or if a large contact-tracing exercise would be needed if the isolate was confirmed as MTB. ${ }^{2}$

Data from all TB cases were routinely collected by the clinical staff on a simple proforma and annual files stored. A retrospective case note audit and review of laboratory records was conducted to supplement information from this contemporaneously collected data. All patients with AFB smear positive clinical samples submitted between January 2002 and December 2006 were identified and included. Data were collected on the timing of smear and culture results, the use of NAAT and their impact on clinical decision making. The appropriateness of referral for NAAT and the proportion of patients with TB screened for HIV infection were determined from the clinical data and stratified according to whether the patient was managed by the multidisciplinary group or by other physicians. Data were entered and analysed in Excel, using simple descriptive statistics. Proportions were expressed as percentages with $95 \%$ confidence intervals (CI) where appropriate and compared using the $\chi^{2}$ test or Fisher's exact test. Continuous variables were compared using the $t$ test. For all analyses, statistical significance was inferred if the $p$ value was below 0.05 .

The study was registered with the hospital's clinical information audit committee and all personal data were stored and analysed in accordance with national guidelines. ${ }^{15}$

\section{RESULTS}

During the study period, 13343 samples were submitted from 6985 patients. Mycobacteria were seen on smears or isolated from the cultures of 651 samples taken from 282 (4\%) of the investigated patients. A total of 123 (44\%) of the 282 patients produced a sample that was AFB smear positive on at least one occasion. Ninety-three (76\%) of these 123 samples were sputum, $13(11 \%)$ were bronchoalveolar lavage samples, 7 $(6 \%)$ were cervical lymph node aspirates and the remainder were from other sites. TB was confirmed by culture in 180 patients (8/180 MDR), by NAAT alone in one patient and other mycobacteria were isolated from the remaining 100 patients (table 1). Two patients were scanty smear positive on single samples but culture negative. One was a lymph node aspirate from a patient previously treated for TB and was NAAT positive 
Table 2 Nucleic acid amplification test results from acid fast bacillus smear positive samples, 2002-2006

\begin{tabular}{|c|c|c|c|c|c|c|c|}
\hline & & & Total & $\begin{array}{l}\text { NAAT indicates } \\
\text { MTB } \\
\text { (n (\%)) }\end{array}$ & $\begin{array}{l}\text { Culture confirms } \\
\text { MTB } \\
\text { (n (\%)) }\end{array}$ & $\begin{array}{l}\text { NAAT indicates } \\
\text { rifampicin } \\
\text { resistance (n (\%)) }\end{array}$ & $\begin{array}{l}\text { Culture indicates } \\
\text { rifampicin } \\
\text { resistance (n (\%) }\end{array}$ \\
\hline \multirow[t]{3}{*}{ Indication for NAAT present } & Sent & Total & 51 & $42(82)$ & 47 (92) & $2(4)$ & $3(6)$ \\
\hline & & $\begin{array}{l}\text { Rifampicin } \\
\text { resistance }^{*}\end{array}$ & 38 & $32(82)$ & $37(97)$ & $2(5)$ & $3(8)$ \\
\hline & & $\begin{array}{l}\text { Rifampicin } \\
\text { resistance }^{*}\end{array}$ & 32 & & $30(94)$ & & $1(3)$ \\
\hline Indication for NAAT not present & & & 36 & $\mathrm{~N} / \mathrm{A}$ & $19(53)$ & $\mathrm{N} / \mathrm{A}$ & 0 \\
\hline Total & & & 123 & $42(34)$ & $98(80)$ & $2(2)$ & $4(3)$ \\
\hline
\end{tabular}

*The principal reason for sending sample for NAAT.

AFB, acid fast bacillus; MDR, multidrug resistant; MTB, Mycobacterium tuberculosis; N/A, not applicable; NAAT, nucleic acid amplification tests.

for $M$ tuberculosis. The other was from a bronchoalveolar lavage in a patient with a clinical and histological diagnosis of bronchial carcinoma. Using culture as the gold standard, the smear sensitivity for $M$ tuberculosis was 98/180 (54\% (95\% CI 47\%, $62 \%)$ ) and specificity was $77 / 102(75 \%$ (95\% CI 66\%, 83\%)).

\section{Use of NAAT}

NAAT were indicated for $87 / 123(71 \%)$ patients and were performed in 51 (59\%). In the remaining 36, NAAT was not performed because it was thought unnecessary or not considered $(n=19)$ or an additional undecontaminated sample was not available $(n=17)$. NAAT was carried out on $44 / 66(67 \%$ (95\% CI 55\%, 77\%)) AFB positive sputum samples in which they were indicated compared with $7 / 21$ (33\% (95\% CI $16 \%$, $55 \%)$ ) non-sputum samples $(\mathrm{p}<0.001)$. The appropriate use of NAAT increased over the study period (fig 1). Between 2002 and 2004, NAAT were indicated in 42 AFB smear positive samples and performed in 16 (38\% (95\%CI 24\%,53\%)). This increased in 2005 and 2006 to $35 / 45$ (78\% (95\% CI 64\%, 88\%)) samples $(\mathrm{p}<0.001)$.

The commonest indication for requesting NAAT was that the patient originated from a TB endemic country where there was

$\square$ Identification and rifampicin resistance detection using NAAT
$\square$ Identification using culture
$\square$ Rifampicin resistance detection using culture

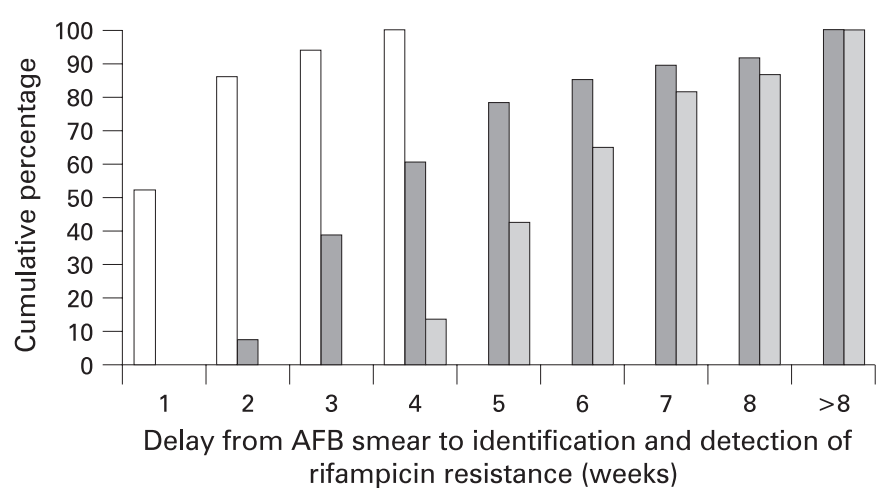

Figure 2 Delay between receipt of acid fast bacillus (AFB) smear positive samples and identification of isolate by nucleic acid amplification tests (NAAT) (in 51 sent for NAAT), identification of the isolate by culture (in all 123 samples) and detection of rifampicin sensitivity (in all 98 samples that grew MTB), 2002-2006. a concern about the possibility of drug resistant infection. A total of 27 (31\%) of the 87 patients in whom NAAT were indicated were HIV positive. However, HIV status was not determined in 20/87 (23\% (95\% CI 15\%, 33\%)) patients in whom NAAT were otherwise indicated and 27/36 (75\% (95\% CI $59 \%, 87 \%)$ ) patients in whom NAAT were not indicated.

\section{Identification and sensitivity results}

Final mycobacterial identification and sensitivity results with a breakdown of NAAT usage are summarised in table 1 and the results of NAAT compared with routine culture in the AFB smear positive samples are shown in table 2. NAAT detected MTB in 41/47 samples in which MTB was subsequently isolated (sensitivity $87 \%$ (95\% CI $75 \%, 95 \%)$ ). In five patients NAAT was negative (in three only scanty AFB were seen on the smear) but MTB was eventually isolated (including one MDR strain), and in the sixth, inhibitors rendered the NAAT result indeterminate. In one further sample, a lymph node aspirate from a patient previously treated for TB, MTB was detected by NAAT but not isolated by culture. The specificity of the NAAT results was $3 / 4(75 \%$ (95\% CI $24 \%, 99 \%))$. MTB was significantly more likely to be isolated from a sample in which NAAT was indicated by the British guidelines compared with those in which it was not indicated $79 / 87$ (91\% (95\% CI 83\%, $96 \%)$ ) vs $19 / 36$ (53\% (95\% CI 37\%, 69\%)); p<0.001). NAAT detected a mutation consistent with rifampicin resistance in two patients, both confirmed by subsequent culture. In one patient with a rifampicin resistant isolate NAAT were negative for MTB. In one further patient with a rifampicin resistant isolate, NAAT were indicated but not sent.

The time to identification of MTB and detection of rifampicin resistance was significantly reduced by the use of NAAT. The mean (SD) time to identification of MTB and detection of rifampicin resistance in patients with NAAT performed was 9.8 (5.9) days compared with 26.0 (10.9) days to identification $(p<0.001)$ and 40.1 (12.9) days to sensitivity test results $(p<0.001)$ using standard methods. For all of the AFB smear positive samples, a total of $86 \%$ of samples were identified by NAAT within 2 weeks compared with $7 \%$ of samples identified using standard methods (fig 2).

\section{Clinical impact of the NAAT result}

The use of NAAT had a clinical impact in 20/51 (39\% (95\% CI $27 \%, 53 \%)$ ) patients for whom it was performed. Three patients who had started TB treatment were able to stop within 2 weeks 
and in one of these cases a major prison contact-tracing exercise was avoided; two of four patients with MDR-TB were identified promptly by NAAT; in three patients, detection of MTB confirmed the need for a hospital contact-tracing exercise; in five previously treated patients, MDR-TB was excluded; and in seven patients for whom there was uncertainty about the diagnosis, TB was confirmed and appropriate treatment continued. In addition, MDR-TB was excluded in 26 patients who originated in TB endemic countries.

If NAAT had been sent from the other 36 patients in which they were indicated, the subsequent culture results suggest it could have had an impact in 8 (22\% (95\% CI 11\%, 38\%)) by detecting one further case of MDR-TB, excluding MDR-TB in two patients previously treated, confirming $\mathrm{TB}$ in two patients and excluding it in three for whom there was diagnostic uncertainty. There could have been further impact by excluding MDR-TB in 26 patients in whom there were risk factors.

If NAAT had also been sent for the 36 patients with AFB positive samples, in whom it was not indicated by the British guidelines, the subsequent culture results suggest that they could have had a clinical influence by stopping unnecessary treatment in 5/35 (14\% (95\% CI 5\%, 29\%)) patients who did not have $\mathrm{TB}$ and a potential clinical impact by providing a rapid confirmation of $\mathrm{TB}$ in a further 19 patients and excluding the diagnosis in 12 .

\section{Impact of the multidisciplinary group}

The multidisciplinary group was convened at the start of 2003. During the study period, $132 / 282$ (47\%) patients with a mycobacterium seen or isolated were managed by the multidisciplinary group. NAAT were indicated and performed in 45/ $66(68 \%$ (95\% CI 56\%, 79\%)) AFB positive samples managed by the multidisciplinary group and 6/21 (29\% (95\% CI 12\%, 50\%)) managed by other physicians $(p=0.001)$. Of the 181 patients diagnosed with TB by culture or NAAT during the study period, $37(20 \%)$ were HIV positive, 78 (43\%) were HIV negative and in $66(36 \%)$ the HIV status was unknown. A HIV test was performed for $95 / 111$ (86\% (95\% CI $78 \%, 91 \%)$ ) of TB culture positive patients managed by the multidisciplinary group but only for $20 / 70$ (29\% (95\% CI 19\%, 40\%)) patients managed by the other physicians $(p<0.001)$

\section{DISCUSSION}

In our region, a relatively high proportion of environmental mycobacterial isolates can confuse the clinical picture and complicate decisions about empirical treatment and contacttracing. In this low prevalence setting for MTB, the sensitivity and specificity of the NAAT were consistent with other studies $^{14}{ }^{16}$ and the NAAT had a direct impact on the clinical management of one-third of AFB smear positive patients in whom it was used. This included patients for whom treatment was changed and situations in which contact-tracing exercises were either commenced or stopped. In addition, the NAAT result was of value in confirming that the correct therapy had been instituted in other patients. Usage of NAAT significantly decreased the time to identification of the mycobacteria and detection or exclusion of rifampicin resistance, allowing prompt alterations in management when needed.

This study shows that the current British guidelines for the use of NAAT in smear positive samples provide a high positive predictive value for detecting MTB and MDR strains. Our data also suggest that extending the indications for performing NAAT on AFB positive samples to include all smear positive samples in low prevalence settings would have additional clinical benefit. In particular, in an area where more than onethird of the mycobacteria isolated are not $M$ tuberculosis, ${ }^{11}$ in the appropriate clinical circumstances rapid exclusion of TB allows patients with AFB positive samples to stop antituberculous treatment.

The results of this study contrast with those from a London teaching hospital where the impact of NAAT was considered to be minimal. ${ }^{16}$ Firstly, this previous study did not look at the benefits of NAAT for the detection of rifampicin resistance. Furthermore, in that setting, an additional two of 19 patients were started on treatment when the NAAT result became available. AFB negative samples were also examined by NAAT but there was no evidence that clinicians were using negative NAAT results to stop treatment. It is possible that the proportion of MTB in AFB smear positive patients is higher in London compared with our setting. If the prior probability that a patient with an AFB positive sample actually has TB is low, as in our patients, the value of NAAT is increased, as our data demonstrate.

A common reason for not sending a sample for NAAT was the need for an undecontaminated sample, requested to reduce the risk of potential contamination from the referring laboratory. On some occasions, however, it was difficult to obtain a further sample; even repeat sputum samples posed logistic difficulties at times. Furthermore, the process of determining whether a patient fulfills the criteria to send a sample can introduce additional delay-mitigated in our study by the multidisciplinary group. Our data imply that the usage of NAAT would be improved and simplified by sending all AFB positive samples and also by allowing a decontaminated sample to be sent if an undecontaminated sample is not quickly and easily obtainable.

One indication for sending a sample for NAAT is knowledge of the patient's HIV status. ${ }^{2}{ }^{5}$ This is also critical for the appropriate management of patients. However, HIV status was not determined for $23 \%$ of the AFB positive patients in whom NAAT were indicated or for $75 \%$ of the AFB positive patients in whom NAAT were not indicated. Overall, HIV tests were not done for one-third of patients with confirmed TB. This is an important gap in the assessment of patients with TB and may reflect a lack of confidence among some physicians in dealing with HIV related issues. The introduction of a multidisciplinary group in our hospital led to a significant increase in HIV testing among patients who were AFB smear positive or with confirmed TB.

Formal costing of the increasing use of NAAT has not been undertaken as part of this study but existing cost effectiveness studies from the UK and overseas indicate that the use of NAAT is overall more cost effective when averted drug treatment, inpatient hospital stays and further investigations are accounted for. ${ }^{17-19}$ The three cases where NAAT results differed from the final identification or sensitivity and the need for a full sensitivity profile imply that standard diagnostic methods still need to be performed.

\section{CONCLUSIONS}

There have been significant clinical benefits from the use of NAAT in this low prevalence setting. The current British guidelines for the use of NAAT have a high positive predictive value when implemented correctly and our data indicate that there would be additional clinical benefit from NAAT being applied to all smear positive clinical samples. A multidisciplinary 


\section{What is already known about this topic}

- Nucleic acid amplification tests allow rapid identification of Mycobacterium tuberculosis, and detection of rifampicin resistance, in AFB positive clinical samples.

- British guidelines include indications for when to use NAAT in clinical practice.

- The appropriateness and clinical impact of these guidelines is not known.

\section{What this study adds}

- A NAAT result had a significant clinical impact in $39 \%$ of the patients with AFB positive samples in which the test was indicated and sent.

- The use of NAAT in all AFB positive samples would have additional clinical benefits.

- A significant number of patients with tuberculosis are still not tested for HIV.

- Weekly review of new mycobacterial diagnoses by a multidisciplinary group may lead to an increase in the appropriate use of NAAT and an improved uptake of HIV testing.

team led to increased appropriate use of NAAT and screening of patients for HIV infection.

Acknowledgements: The staff of the Royal Liverpool University Hospital Department of Microbiology, and the Regional Centre of Mycobacteriology, Newcastle and the United Kingdom Health Protection Agency National Mycobacterial Reference Unit. We are grateful for the comments on the manuscript by Dr Timothy Brown, Health Protection Agency National Mycobacterial Reference Unit and Dr Richard Cooke, University Hospital Aintree, Liverpool, UK,

Competing interests: None.

Ethics approval: The study is a registered hospital audit and ethical approval was not required.

\section{REFERENCES}

1. Health Protection Agency. Tuberculosis: latest epidemiological data. http:// www.hpa.org.uk/infections/topics az/tb/data menu.htm 2006 laccessed 6 February 2008).

2. National Collaborating Centre for Chronic Conditions. Tuberculosis: clinical diagnosis and management of tuberculosis and measures for its prevention and control. London: Royal College of Physicians, 2006.

3. Caws M, Drobniewski FA. Molecular techniques in the diagnosis of Mycobacterium tuberculosis and the detection of drug resistance. Ann N Y Acad Sci 2001;953:13845.

4. Drobniewski FA, Hoffner S, Rusch-Gerdes S, et al. Recommended standards for modern tuberculosis laboratory services in Europe. Eur Respir J 2006;28:903-9.

5. Control and prevention of tuberculosis in the United Kingdom: code of practice 2000. Joint Tuberculosis Committee of the British Thoracic Society. Thorax 2000;55:887901

6. Aziz MA, Wright A, Laszlo A, et al. Epidemiology of antituberculosis drug resistance (the Global Project on Anti-tuberculosis Drug Resistance Surveillance): an updated analysis. Lancet 2006;368:2142-54.

7. Gandhi NR, Moll A, Sturm AW, et al. Extensively drug-resistant tuberculosis as a cause of death in patients co-infected with tuberculosis and HIV in a rural area of South Africa Lancet 2006;368:1575-80.

8. Telenti A, Imboden P, Marchesi F, et al. Detection of rifampicin-resistance mutations in Mycobacterium tuberculosis. Lancet 1993;341:647-50.

9. Goyal M, Shaw RJ, Banerjee DK, et al. Rapid detection of multidrug-resistant tuberculosis. Eur Respir J 1997;10:1120-4.

10. Drobniewski FA. Diagnosing multidrug resistant tuberculosis in Britain. Clinical suspicion should drive rapid diagnosis. BMJ 1998;317:1263-4.

11. Corless JA, Stockton PA, Davies PD. Mycobacterial culture results of smear-positive patients with suspected pulmonary tuberculosis in Liverpool. Eur Respir J 2000;16:976-9.

12. Antoine D, Maguire H, Story A. Epidemiology and response to the growing problem of tuberculosis in London. Euro Surveill 2006;11:25-8.

13. Home Office. Immigration and Asylum Act. London: The Stationery Office, 1999 http://www.opsi.gov.uk/acts/acts1999/19990033.htm (accessed 6 February 2008).

14. Sam IC, Drobniewski F, More P, et al. Mycobacterium tuberculosis and rifampin resistance, United Kingdom. Emerg Infect Dis 2006;12:752-9.

15. Department of Health. The Caldicott Committee Report on the Review of PatientIdentifiable Information. London: Department of Health, 1997.

16. Conaty SJ, Claxton AP, Enoch DA, et al. The interpretation of nucleic acid amplification tests for tuberculosis: do rapid tests change treatment decisions? $J$ Infect 2005;50:187-92.

17. NHS Health Technology Assessment Programme. The clinical and costeffectiveness of diagnostic tests for the detection of mycobacterial infection, 2004. www.ncchta.org/project (accessed 6 February 2008).

18. Drobniewski FA, Watterson SA, Wilson SM, et al. A clinical, microbiological and economic analysis of a national service for the rapid molecular diagnosis of tuberculosis and rifampicin resistance in Mycobacterium tuberculosis. J Med Microbiol 2000;49:271-8.

19. van Cleeff M, Kivihya-Ndugga L, Githui W, et al. Cost-effectiveness of polymerase chain reaction versus Ziehl-Neelsen smear microscopy for diagnosis of tuberculosis in Kenya. Int J Tuberc Lung Dis 2005;9:877-83. 